\title{
Hydraulic supports for polishing TMT M3MP
}

\author{
Haifei $\mathrm{Hu}^{* a}$, Erhui Qi ${ }^{\mathrm{a}}$, Glen Cole ${ }^{\mathrm{b}}$, Haixiang $\mathrm{Hu}^{\mathrm{a}, \mathrm{c}}$, Xiao Luo ${ }^{\mathrm{a}}$, Virginia Ford ${ }^{\mathrm{b}}$, Xuejun Zhang ${ }^{\mathrm{a}}$ \\ ${ }^{a}$ Changchun Institute of Optics, Fine Mechanics and Physics, Chinese Academy of Sciences, \\ Changchun 130033, Jilin, China \\ ${ }^{\mathrm{b}}$ Thirty Meter Telescope Observatory Corporation, Pasadena, CA 91105, USA \\ ${ }^{c}$ University of Chinese Academy of Sciences, Beijing 100049, China
}

\begin{abstract}
For polishing the ultra-thin TMT M3MP, a polishing support system with 18 hydraulic supports (HS) is introduced. This work focuses on the designing and testing of these HSs. Firstly the design concept of HS system is discussed; then mechanical implementation of the HS structure is carried out, with special consideration of fluid cycling, work pressurization and the weight component. Afterward the piping installation and the de-gas process for the working fluid are implemented. Pressurization and stiffness are well checked before system integration for the single HS unit. Finally the support system is integrated for the polishing process.
\end{abstract}

Keywords: Ultra-thin mirror, hydraulic support, de-gas process, pressurization, stiffness

\section{INTRODUCTION}

TMT is one of the world's largest telescopes and has attracted staff from over five countries to join in. CIOMP has been involved in TMT project since 2012, and is responsible to fabricate the tertiary mirror system, keeping the mirror surface's root mean square of slope values (SlopeRMS) bellow 0.89 micro-radians $(\mu \mathrm{rad}) .{ }^{1}$ The M3 Mirror (M3M) is made of Zerodur and is an elliptical flat mirror with dimensions $3594 \times 2536 \times 100 \mathrm{~mm}^{3}$ having an aspect ratio of 35 . The required SlopeRMS is rather hard to obtain. In addition, the requirement for the SlopeRMS value was assumed to apply with the mirror set upon its axial support system facing upwards in a 1 gravity environment, so that support print-through is polished out. For feasibility study and reducing the risk, a prototype (M3P) scaled by $25 \%$ has been fabricated.

For fabricating large mirrors, people usually use a polishing support system, different from the structural support system, to restrain the magnitude of "Print-through effect" and gravity off-loading deflection. ${ }^{2-6}$ For the prototype mirror (M3P), a custom polishing support to achieve polishing out print-through was developed and used.

\section{CONCEPTUAL DESIGN}

\subsection{Scaling mirror dimensions}

This thin mirror's deflection could be roughly decided by analytical solution from theory of thin plates. According to Timoshenko's theory, for circular plate with clamped edges and bearing uniform load, the maximum deflection is at the center of the plate and could be expressed as ${ }^{7}$

$$
W=\frac{q a^{4}}{64 D},
$$

where $w$ is the deflection, $q$ is the uniform load, $a$ is the radius of the circular plate, and $D$ is the Flexural rigidity. $D$

could be expressed as $D=\frac{E h^{3}}{12\left(1-v^{2}\right)}$, where $E$ is Young's modulus, $v$ is Poisson's ratio and $h$ is the plate thickness.

When the mirror is loaded by gravity, $q=\rho g h$, where $\rho$ is the material density and $g$ is the gravity acceleration. So equation (1) could be rewritten as

\footnotetext{
*E-mail: huhf@ciomp.ac.cn, Tel: +864318670 8687

AOMATT 2016: Large Mirrors and Telescopes, edited by Myung Kyu Cho, Bin Fan, Proc. of SPIE Vol. 9682, $968208 \cdot$ (c) 2016 SPIE · CCC code: $0277-786 X / 16 / \$ 18 \cdot$ doi: 10.1117/12.2245642
} 


$$
W=\frac{3\left(1-v^{2}\right) \rho g a^{4}}{16 E h^{2}} .
$$

And the corresponding slope could be derived by

$$
S=\frac{\partial w}{\partial a}=\frac{3\left(1-v^{2}\right) \rho g a^{3}}{4 E h^{2}} .
$$

It's seen from equation (3), for keeping $s$ almost the same, if the aperture $a$ is scaled by $1 / 4$, the thickness $h$ should be scaled by $1 / 8$. As M3's dimensions are $a=3594 \mathrm{~mm}, b=2536 \mathrm{~mm}$ and $h=100 \mathrm{~mm}$, the prototype's dimensions are scaled to $a^{\prime}=898.5 \mathrm{~mm}, b^{\prime}=634 \mathrm{~mm}$ and $h^{\prime}=12.5 \mathrm{~mm}$ respectively.

For deciding the number of support points $N$ needed to limit the mirror surface deflection between discrete supports to some tolerable value $\delta$, there's a simplified equation in SI unit system written as following ${ }^{8}$

$$
N=\frac{3 L^{2}}{8 h} \sqrt{\frac{\rho g}{E \delta}},
$$

where $N$ is the minimum number of support points, $L$ stands for the largest dimension of the aperture; other parameters are as previously defined. For M3P, $L=a^{\prime}=898.5 \mathrm{~mm}, h=h^{\prime}=12.5 \mathrm{~mm}, \rho=2.53 \mathrm{e}-9 \mathrm{t} / \mathrm{mm}^{3}, E=9.03 \mathrm{e} 4 \mathrm{MPa}, \delta=6.33 \mathrm{e}-5 \mathrm{~mm}$, the corresponding $N$ could be calculated as $N=51$. For whiffle-tree implementation, a number of 54 for $N$ is chosen.

\subsection{Layout optimization}

The FEA model of M3P for the polishing support system is shown in Fig 1. Due to symmetry, a quadrant of the mirror was developed, and a polishing pressure of $1 \mathrm{kPa}$, whose total action force is assumed to be equally born by the 18 polishing supports, is applied to the reflecting surface. In the model, there are 19 hard points, shown in green triangles, which include 14 points representing polishing support points and the remaining 5 points representing mirror axial support points. When polishing the mirror, the polishing supports rise to contact the back side of the mirror for balancing polishing pressure. The gravity sag of the glass between the axial supports is maintained. Symmetric displacement conditions are applied to the nodes located on axes. And the node centered on the ellipse is restrained in $\mathrm{Z}$ direction for FE solution consideration. For updating the moving boundary conditions, one just needs to change the location of these hard points.

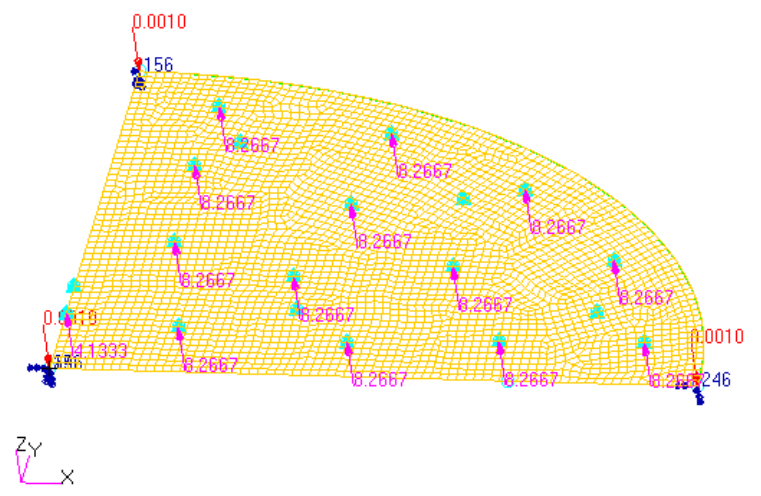

Fig 1 Conceptional model for M3MP polishing support

Essentially here layout optimization of mirror supports mainly refers to a reverse problem for finding locations where forced boundary conditions should be imposed to minimize the deflection of the surface. Layout optimization model of $n$ supports could be described as 


$$
\begin{array}{ll}
\text { Find } & P_{i}\left(x_{i}, y_{i}\right), i=1,2, \ldots, n \\
\text { Minimize } & \operatorname{RMS}\left(\mathrm{d} z_{k}\right), k=1,2, \ldots, N_{\mathrm{G}}, \\
& \text { and } \operatorname{SlopeRMS}\left(\mathrm{d} z_{k}\right), \\
\text { Subject to } & P\left(x_{i}, y_{i}\right) \in \Omega \\
& \text { and }\left|P_{i} P_{j}\right|>D, i \neq j
\end{array}
$$

where $P_{i}\left(x_{i}, y_{i}\right)$ represents the location of support $i, \mathrm{~d} z_{k}$ is the difference between the actual position and the ideal position of grid $k, N_{G}$ is the number of surface grids, $\Omega$ is the aperture of the mirror, and $\left|P_{i} P_{j}\right|>D$ implies the fact that the distance between two supports should be larger than a certain distance of $D$, e.g., the largest diameter of the support structure. The PV and RMS are calculated by Zernike polynomials fitting, and SlopeRMS is calculated using equation:

$$
\text { SlopeRMS }=\sqrt{\left(\frac{1}{(N-1)(M-1)}\right) \sum_{i=1}^{N-1} \sum_{j=1}^{M-1}\left[\left(\frac{S_{i+1, j}-S_{i, j}}{\mathrm{~d} x}\right)^{2}+\left(\frac{S_{i, j+1}-S_{i, j}}{\mathrm{~d} y}\right)^{2}\right]},
$$

where $N$ is the number of sample grids in $x$ direction, $M$ is the number of sample grids in $y$ direction, $S_{i j}$ is the figure difference of grid $(i, j), \mathrm{d} x$ and $\mathrm{d} y$ are the sample distances.

Optimized indexes $\delta_{\mathrm{PV}}, \delta_{\mathrm{RMS}}$ and $\delta_{\text {SlopeRMS }}$ are shown in Tab 1. From Tab 1, it's seen that through layout optimization of support points, $\delta_{\mathrm{PV}}$ is reduced from to $1756 \mathrm{~nm}$ to $80.6 \mathrm{~nm}$, scaled by $4.6 \% ; \delta_{\mathrm{RMS}}$ is reduced from to $417 \mathrm{~nm}$ to $12.7 \mathrm{~nm}$, scaled by $3.0 \% ; \delta_{\text {SlopeRMS }}$ is reduced from to $4.56 \mu \mathrm{rad}$ to $0.71 \mu \mathrm{rad}$, scaled by $15.6 \%$. The optimized $\delta_{\text {RMS }}$ is about $\lambda / 50$, and the optimized $\delta_{\text {slopeRMs }}$ is within allowed $0.8 \mu \mathrm{rad}$.

Tab 1 Results summary of initial scheme and optimized scheme

\begin{tabular}{ccc|ccc}
\hline \multicolumn{3}{c|}{ Initial layout } & \multicolumn{3}{c}{ Optimized layout } \\
\hline $\boldsymbol{\delta}_{\mathrm{PV}} / \mathbf{n m}$ & $\boldsymbol{\delta}_{\mathrm{RMS}} / \mathbf{n m}$ & $\boldsymbol{\delta}_{\text {SlopeRMS }} / \boldsymbol{\mu r a d}$ & $\boldsymbol{\delta}_{\mathrm{PV}} / \mathbf{n m}$ & $\boldsymbol{\delta}_{\mathrm{RMS}} / \mathbf{n m}$ & $\boldsymbol{\delta}_{\text {SlopeRMS }} / \boldsymbol{\mu r a d}$ \\
\hline 1756 & 417 & 4.56 & 80.6 & 12.7 & 0.71 \\
\hline
\end{tabular}

The distribution of surface grids' displacements and slopes are shown in Fig 2.

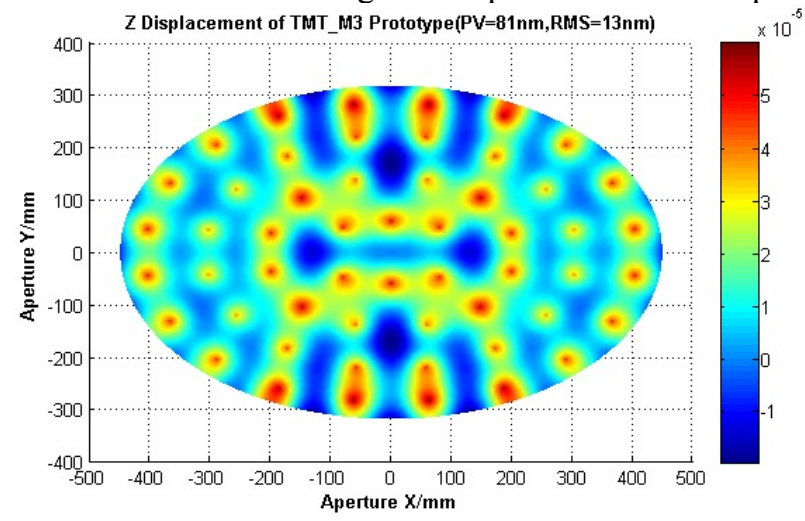

(a) Displacement distribution of optimized scheme

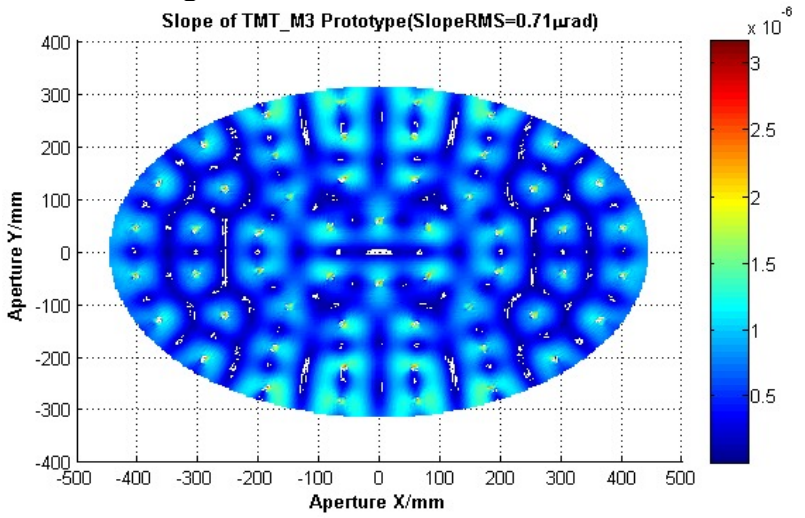

(b) Slope distribution of optimized scheme

Fig 2 Figure accuracy comparison of initial scheme and optimized scheme

From Fig 2 it's seen that the optimized layout makes the mirror's deformation much more uniform and makes the magnitude of displacements much smaller. Layout optimization brings a significant improvement for the mirror's rigidity. 


\section{MECHANICAL IMPLEMENTATION}

The implementation of the hydraulic support are divided into 3 steps: (1) design of the support system and the single hydraulic unit; (2) stiffness test of the units; and (3) the system integration.

\subsection{Design of the support system and the single hydraulic unit}

The mechanical implementation for the manufacturing support system is illustrated in Fig 3, where the lateral support system is also illustrated. There are 54 hydraulic polishing supports divided into 3 groups, each of which is supported by 6 equal-force hydraulic supports. Each hydraulic support controls a load spreader that links 3 pads attached on the mirror's rear side. The 3 pads are designed to average the force of each hydraulic support.

For each single hydraulic support unit, the mechanical design is shown in Fig 4 (a). The input and the output pipes are linked to a sphere valve. So it could be seen that the input pipe, the static piston, the diaphragm, the cover and the output pipe forms a narrow space for the work fluid to fill in. The lateral limit board provides a free motion of about $8 \mathrm{~mm}$ for the floating components. A plastic seal cushion fastened by the seal bolt produces a good pressurization. Also the seal bolt acts as the lateral limit when not working, so it could holding the mirror at a high precision after the integration of the manufacturing support system. The fluid used for working flow is glycerin, as it is viscous and has a high bulk modulus.

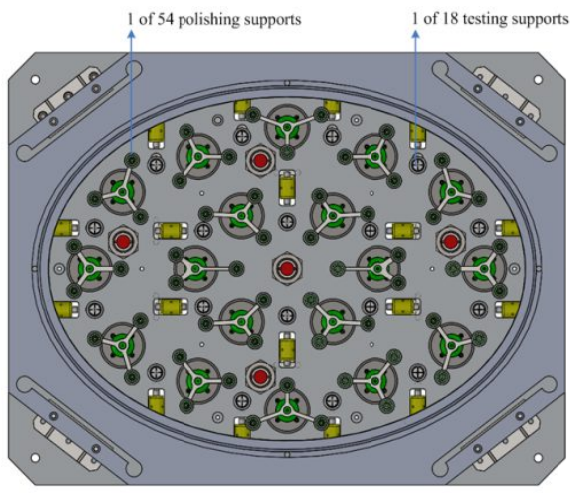

(a) $2 \mathrm{D}$ view

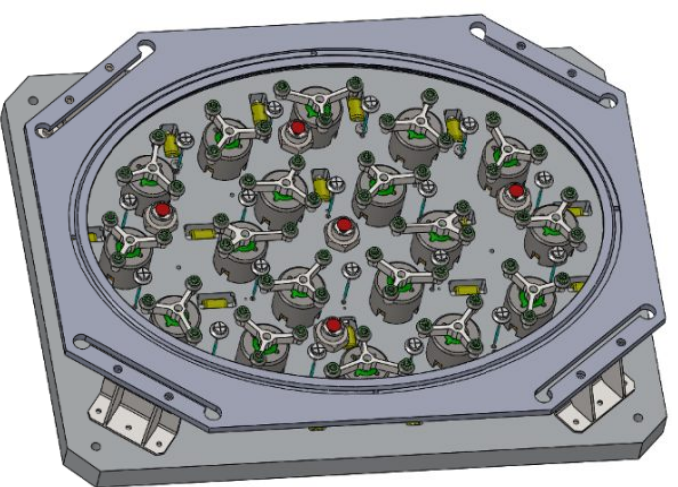

(b) 3D view

Fig 3 Mechanical implementation for manufacturing TMTM3P, 54 support points for polishing and 18 points for testing

(2) Stiffness test of the units

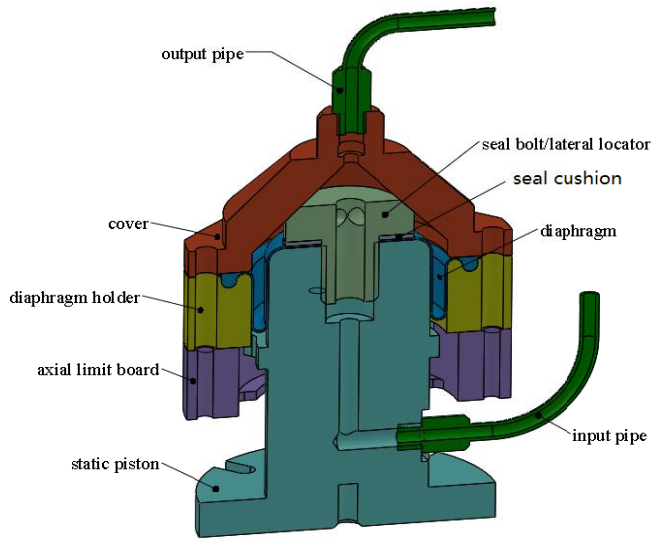

(a) Cross-section of the support

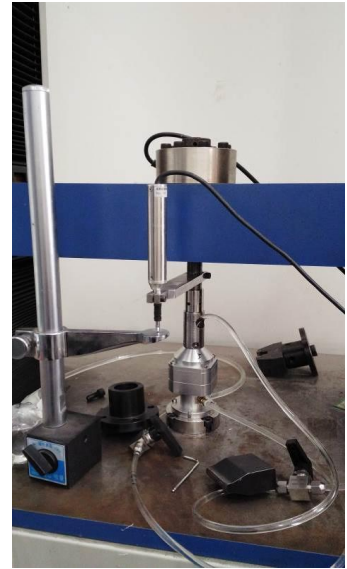

(b) Stiffness test

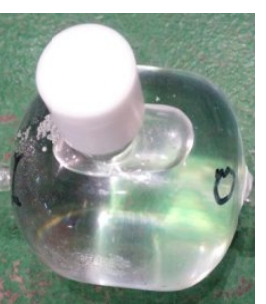

(c)Air buffer bottle

Fig 4 Mechanical design and stiffness test of a single hydraulic support unit

Stiffness tests of the hydraulic supports were performed using a Chinese National Standard Machine for mechanical testing, shown in Fig 4(b). We load the support toward $200 \mathrm{~N}$, approximating the polishing load of $20 \mathrm{~kg}$; record the force $F$ and the corresponding displacement $u$ of the support; and compute the stiffness k by $k=F / u$. If the curve is not straight enough, we use $k=\mathrm{d} F / \mathrm{d} u$ for a specific region. Most $F-u$ curves are very close to linear. Each hydraulic support was tested least 3 times to improve the test accuracy.

To achieve higher support stiffness, the volume of air bubbles must be minimized, so a buffer bottle is used, shown in 
Fig 4 (c). Most of the air bubbles that are formed during piping will stored in this bottle, which contributes a volume of purer glycerin to the support.

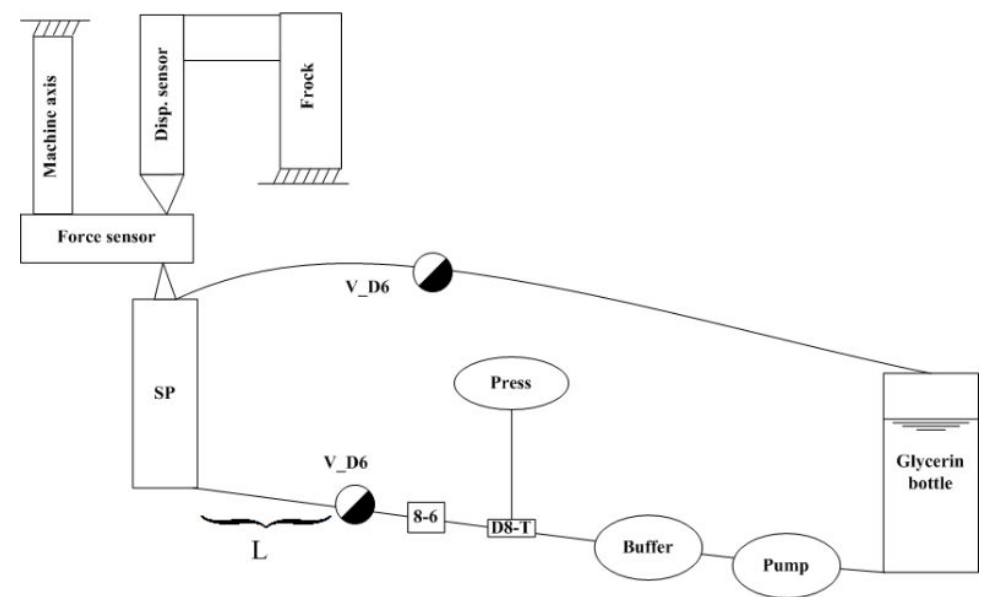

Fig 5 Piping cycle for testing the stiffness

Piping cycle for testing the support unit's stiffness is shown in Fig 5. During the test, we found that the pipe length affects the stiffness significantly, so in the piping cycle the length expressed by $L$, of the input pipe was changed to equalize all stiffness values of the supports.

The stiffness values at certain input pipe length for the used 18 supports are listed in Tab 2 in ascending order. The minimum stiffness is $800 \mathrm{~N} / \mathrm{mm}$ for the support numbered 20, and the maximum is $1188 \mathrm{~N} / \mathrm{mm}$ for the support numbered 11.

Tab 2 Stiffness summary of the 18 supports used

\begin{tabular}{|c|c|c|c|}
\hline Rank No. & SP No & $\mathbf{L}(\mathbf{c m})$ & $\mathbf{S}(\mathbf{N} / \mathbf{m m})$ \\
\hline 1 & 20 & 77 & 800 \\
\hline 2 & 1 & 60 & 804 \\
\hline 3 & 12 & 56 & 807 \\
\hline 4 & 5 & 120 & 821 \\
\hline 5 & 8 & 70 & 826 \\
\hline 6 & 7 & 60 & 837 \\
\hline 7 & 4 & 60 & 850 \\
\hline 8 & 2 & 60 & 881 \\
\hline 9 & 14 & 60 & 893 \\
\hline 10 & 13 & 85 & 896 \\
\hline 11 & 17 & 60 & 906 \\
\hline 12 & 6 & 60 & 921 \\
\hline 13 & 10 & 60 & 974 \\
\hline 14 & 9 & 60 & 1027 \\
\hline 15 & 16 & 63 & 1071 \\
\hline 16 & 15 & 70 & 1092 \\
\hline 17 & 18 & 72 & 1135 \\
\hline 18 & 11 & 60 & 1188 \\
\hline
\end{tabular}

\subsection{Hydraulic system integration}

According to their stiffness, all 18 supports are integrated into 3 groups, surrounded by 3 bold dark curves shown in Fig 6(a). Group division is also shown in Tab 2 by different color. In detail, the support tick numbers for group 1 are 12, 7, 8, 2,14 and 6; the support tick numbers for group 2 are 1,20,5, 4, 13 and 17; the support tick numbers for group 3 are 16, $9,10,11,15$ and 18 . 


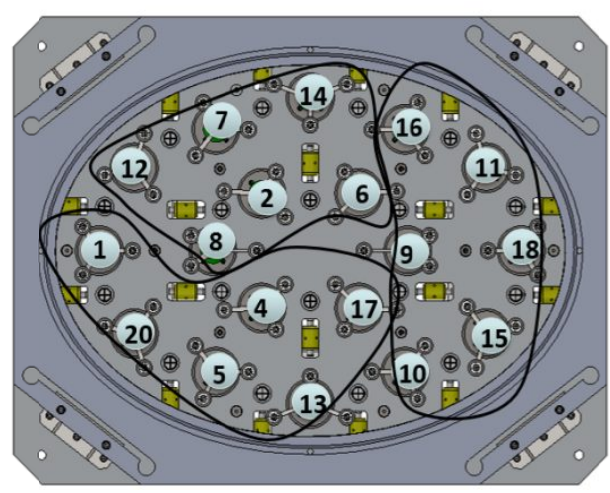

(a) Group division

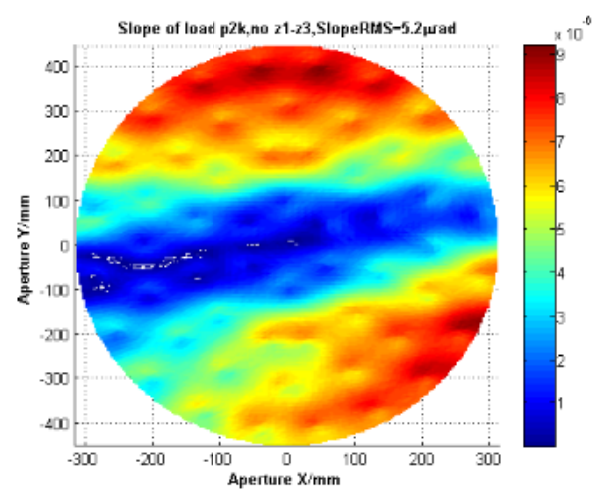

(b) Performance of real support

Fig 6 System integration and performance evaluation

Support performance evaluation is carried out after system integration, as shown in Fig 6(b). The FE model uses 18 springs with the tested stiffness supporting the mirror, and the polishing pressure is $2 \mathrm{kPa}$, resulting in $5.2 \mu \mathrm{rad}$ SlopeRMS, with a mean of $2.6 \mu \mathrm{rad} / \mathrm{kPa}$. This difference is mainly caused by the difference of stiffness. To overcome those stiffness difference, during operation all input valves are opened within a group to get closer stiffness.

\section{CONCLUSIONS}

The proposed support scheme is obtained through layout optimization of support points. The designed SlopeRMS for print-through is about $0.71 \mu \mathrm{rad}$, lower than allowed $0.8 \mu \mathrm{rad}$. Whereas the performance of the real stiffness is worse to $2.6 \mu \mathrm{rad}$, which is not as good as designed, it's very necessary to open all input valves to get closer stiffness. Actually if the space between the mirror and the polishing pad is larger than $10 \mu \mathrm{m}$, which is usually the minimum size of polishing powder, material removal rate could still be kept high enough. Also only the testing support and the fabrication error contribute to the mirror's surface figure accuracy, so the polishing support system serves well till the mirror is polished to the required precision.

But it is intended that further measures should be taken to get closer stiffness for M3M because uniform support stiffness will unburden the processing algorithm much.

\section{ACKNOWLEDGEMENT}

This work is financially supported by Natural Science Foundation of China No.61210015 and Chinese National 973 program No.2011CB013205.

\section{REFERENCES}

[1] TMT Group, “Tertiary Mirror Surface Figure Specification,” TMT.OPT.SPE.12.001.CCR01, 12 (2014).

[2] Smith, WS, "Manufacturing and Testing an 8.3 meter Astronomical Mirror," Proc. of SPIE 3782, 157-168 (1999).

[3] Martin, HM, Allena, RG, Cuerdena, B, et al. "Manufacture of the second $8.4 \mathrm{~m}$ primary mirror for the Large Binocular Telescope," Proc. of SPIE 6273, 62730C (2006).

[4] Schipani, P, Capaccioli, M, 'Orsi, S D, et al, "The VST active primary mirror support system," Proc. of SPIE 7739, 773931 (2010).

[5] Kaercher, HJ, Eisentraeger, P and Süß, M, “Mechanical principles of large mirror supports," Proc. of SPIE 7733, 773320 (2010).

[6] Martin, HM, Callahan, SP, Cuerden, B, et al, "Active supports and force optimization for the MMT primary mirror," Proc. of SPIE 3352, 412-423 (1998).

[7] Timoshenko, S and Woinowsky-Krieger, S, [Theory of plates and shells], McGraw-Hill Book Company Inc., Singapore, 55 (1959).

[8] Yoder, PR, [Opto-Mechanical Systems Design], SPIE Press, Bellingham, 505 (2006). 